\title{
COMUNICACIÓN
}

\section{Parásitos intestinales en una comunidad Amerindia, Costa Rica}

\author{
FRANCISCO HERNÁNDEZ-CHAVARRÍA* y MARÍA FERNÁNDA MATAMOROS-MADRIGAL*
}

\section{INTESTINAL PARASITES IN AN AMERINDIAN COMMUNITY: COSTA RICA}

In Costa Rica there were three national surveys of intestinal parasitism (1966, 1982, and 1996) that showed dramatic reduction of the prevalence of Trichuris, Ascaris, and Necator/Ancylostoma (hookworms); however, these nematodes persist with high prevalence in low socio-economically groups, as the squatter settlements around the cities; however, it is possible that pour Amerindian communities with substandard housing condition also suffer the burden of intestinal parasites. For this reason, the present study was planted in a rural disperse Amerindian community from the South of Costa Rica. 45 fecal samples were collected from children under 15 years old, and were processed according a modification of the Baermann method, which also permits the observation of other parasites beyond Strongyloides. $38(84 \%)$ of the analyzed samples were positive for at least one parasite. The prevalence for nematodes was Ascaris (36\%), hookworms (22\%) Enterobius (4\%), and Trichuris (2\%). For protozoa was Endolimax nana (33\%), Entamoeba coli (27\%), and Entamoeba histolytica/dispar $(11 \%)$. These data are the reflect of the inadequate sanitation conditions of this community.

Key words: Intestinal parasites, Survey, Baermann method, Amerindian community.

\section{INTRODUCCIÓN}

En Costa Rica se han realizado tres encuestas nacionales sobre parasitismo intestinal; la primera la realizó el Instituto de Nutrición para Centro América y Panamá en $1966^{1}$, y las otras dos se realizaron como colaboración entre el Ministerio de Salud y la Universidad de Costa Rica, una en $1982^{2}$ y la otra en $1996^{3}$. Estos tres estudios mostraron un dramático descenso de la prevalencia de helmintiasis, especialmente en Ascaris, Trichuris y Necator/Ancylostoma (uncinarias); que globalmente presentaban una prevalencia cercana al 50\% en 1966 y para 1982 había descendido a menos del 5\% y para 1996 a menos de un $3 \%$. Pero ese cambio drástico no se refleja en comunidades marginales, donde el hacinamiento, la falta de infraestructura para la eliminación adecuada de aguas negras y la pobreza se confabulan para mantener una prevalencia de parasitismo intestinal tan alta como la que experimentaba el país décadas atrás ${ }^{4-6}$. Por otra parte, en las tres encuestas nacionales el análisis de helmintos intestinales se realizó mediante la técnica de recuento de huevos de Stoll, lo que permitió comparar los resultados de los tres estudios; sin embargo, dada la brusca disminución de la prevalencia de helmintiasis, para la tercera

\footnotetext{
* Facultad de Microbiología, Universidad de Costa Rica, San José, Costa Rica. 2. Centro de Investigación en Estructuras Microscópicas (CIEMic) Universidad de Costa Rica, San José, Costa Rica.

* hchavarr@cariari.ucr.ac.cr
} 
encuesta también se analizaron las muestras mediante el método de Kato y dado que las muestras estaban preservadas en formalina, posteriormente se analizaron adicionalmente por examen en directo; sin embargo, no se buscó específicamente Strongyloides; lo que posiblemente explique el porqué la prevalencia de este parásito fue de sólo el $0,1 \%$.

Por otra parte, las comunidades Amerindias costarricenses representan grupos de población marginados del avance económico, social y tecnológico del resto del país, principalmente por sus condiciones de comunidades rurales dispersas $\mathrm{y}$ en algunos casos localizadas en áreas geográficas de difícil acceso. Por ello, es importante evaluar la prevalencia de parásitos intestinales en estos grupos de población y el objetivo de esta investigación fue la evaluación de la prevalencia de $S$. stercoralis mediante análisis de muestras utilizando una modificación del método de Baermann ${ }^{8}$. Aunque la adaptación de este método fue realizada para buscar larvas de $S$. stercoralis, también, actúa como método de concentración por gravedad para quistes de protozoos y huevos de nematodos, lo cual se refleja en el hallazgo de algunos de estos parásitos en muestras que por el examen directo han resultado negativas ${ }^{9}$; no obstante, el citoplasma de los quistes de Giardia tiende a entrar en lisis por lo cual resulta difícil de detectar este protozoo en este tipo de preparaciones.

En este informe se presenta los hallazgos de los análisis parasitológicos de las muestras de heces de un grupo de niños de una población Amerindia del Sur de Costa Rica, cuyo estudio se realizó mediante el método de Baermann modificado.

\section{MATERIAL Y MÉTODOS}

Aprovechando la actividad de extensión social del grupo de odontología de la clínica de Coto Brus (Sur de Costa Rica), que realizan visitas a comunidades indígenas de la región, se planteó un estudio parasicológico en la comunidad Guaymi de Altos de Conte (Golfito). Se colectó un total de 45 muestras de heces de niños menores de 15 años. De cada muestra se montó la adaptación del método de Baermann ${ }^{8}$, la cual brevemente consiste en sustituir el embudo del método estándar por un tubo de 16×100 mm, en el cual se hace una suspensión de heces en solución salina; se tapa con un tapón de hule perforado, al cual se le coloca una punta de micropipeta y este tubo se invierte sobre otro con unos $8 \mathrm{ml}$ de solución salina, que a su vez se coloca en un baño a $37^{\circ} \mathrm{C}$ por un mínimo de 2 horas. Dado que en la comunidad no había suministro de energía eléctrica se improvisó el baño de incubación, con un recipiente plástico rectangular de 4 litros de capacidad, al cual se le cortó una de las caras laterales y se llenó con agua a $\mathrm{ca} .37^{\circ} \mathrm{C}$, donde se colocó una gradilla de alambre con los dispositivos del Baermann. Se dejó en reposo toda la noche y al día siguiente se agregó $1 \mathrm{ml}$ de formalina al $40 \%$ al tubo inferior, se tapó herméticamente y se transportó al laboratorio del Centro de Investigación en Estructuras Microscópicas de la Universidad de Costa Rica, donde aproximadamente una semana más tarde se centrifugaron los tubos y se analizó el sedimento.

\section{RESULTADOS}

De las 45 muestras analizadas se encontraron parásitos en 38 , lo que equivale a un $84 \%$. La mayoría de las muestras presentaban una cantidad abundante de parásitos, ya fuese huevos de nematodos o quistes de protozoos. Se diagnosticaron $2(4 \%)$ casos con S. stercoralis; además, se encontró una prevalencia porcentual de Ascaris y uncinarias de $36 \%$ y $22 \%$, respectivamente; en tanto, la prevalencia de Trichuris fue solo del $2 \%$ y la de Enterobius fue del $4 \%$.

En cuanto protozoos los más frecuentes fueron Endolimax nana y Entamoeba coli con frecuencias de $33 \%$ y $27 \%$, respectivamente; además, hubo 5 casos de Entamoeba histolytical dispar. En la Tabla 1 se muestra la totalidad de los parásitos intestinales diagnosticados.

\section{DISCUSIÓN}

El primer hallazgo que llama la atención en esta comunidad es la alta prevalencia de parásitos intestinales, especialmente en cuanto a helmintos y en éstos, el otro resultado relevante es la baja prevalencia de Trichuris, dado que en los estudios realizados en Costa Rica, la frecuencia informada de los tres geohelmintos más frecuentes siempre coloca a Trichuris de primero, luego a Ascaris y finalmente a las uncinarias; por ejemplo, en la 
Tabla 1. Parásitos intestinales en una comunidad Amerindia, Sur de Costa Rica $(n=45)$

\begin{tabular}{lrr} 
Parásito & $\mathbf{N}^{\mathbf{0}}$ & $\mathbf{( \% )}$ \\
\hline Ascaris & 15 & $(36)$ \\
Necator/Ancylostoma & 11 & $(24)$ \\
Trichuris & 1 & $(2,2)$ \\
Strongyloides & 2 & $(4)$ \\
Enterobius & 2 & $(4)$ \\
Giardia duodenalis & 2 & $(4)$ \\
Entamoeba coli & 12 & $(27)$ \\
Entamoeba histolytica/dispar & 5 & $(11)$ \\
Endolimax nana & 15 & $(33)$ \\
Total & 38 & $(84)$ \\
\hline
\end{tabular}

encuesta de 1996 esos datos fueron de 3,6\%, $2 \%$ y $0,6 \%$ respectivamente ${ }^{3}$. Además, Enterobius solo se diagnostica esporádicamente. Por otra parte, la prevalencia encontrada de $S$. stercoralis resulta relativamente alta, dada que en estudios previos, en poblaciones a riesgo para esta parasitosis, como son alcohólicos o ancianos la prevalencia encontrada ha sido de $6 \%^{10}$ y $2 \%{ }^{11}$, respectivamente. En este tipo de análisis, dado que las muestras se incubaron durante al menos 12 horas, inicialmente a $c a 37^{\circ} \mathrm{C}$ y luego a temperatura ambiente, es importante la discriminación entre larvas de Strongyloides y de uncinarias (Necator/Ancylostoma), pues los huevos de estos últimos parásitos podrían liberar las larvas.

Por otra parte, la adaptación del método de Baermann empleado ha permitido diagnosticar parásitos en muestras de heces, donde el examen directo fue negativo, lo cual incluye a Trichuris, por lo tanto, es poco probable que la baja frecuencia de este nematodo en la población estudiada se deba a deficiencia en la metodología empleada; lo que si podría estar ocurriendo con la prevalencia de Giardia, dado que los quistes de este protozoario aparecen extremadamente claros, con el citoplasma aparentemente en lisis y la formalina no es el fijador idóneo para estos quistes; por lo tanto, es posible que en esta población la prevalencia de Giardia sea mucho mayor a la informada aquî́.

Entre las razones que explican el alto grado de parasitismo intestinal en esta población están la falta de agua potable, que obliga a los habitantes de esta comunidad a extraer el agua de ríos y quebradas, lo cual aunado a la falta de letrinas empeora la situación; por otra parte, muchos de los niños no usan zapatos lo que favorece la infección con uncinarias y Strongyloides.

Finalmente, a pesar de ser una comunidad, cuyas casas están muy dispersas en el bosque, muestran hacinamiento familiar debido a que en general son familias numerosas habitando en casas pequeñas de una o dos habitaciones. Estas características se confabulan para favorecer el parasitismo intestinal detectado en esta comunidad.

En Costa Rica se han realizado 3 encuestas nacionales de parasitismo intestinal (1966, 1982, 1996), que mostraron una reducción dramática de la prevalencia de Trichuris, Ascaris, y Necatorl Ancylostoma (uncinarias); no obstante, estos nemátodos persisten con una alta prevalencia en grupos con bajos niveles socioeconómicos, como los asentamientos precarios alrededor de las ciudades: por lo tanto, es posible que en comunidades Amerindias con bajos estándares de también sufran el impacto del parasitismo intestinal. Por esta razón, se planteo el presente studio en una comunidad Amerindia dispersa el sur de Costa Rica. Se colectaron 45 muestras de heces de niños menores de 15 años de edad y se procesaron de acuerdo con una modificación del método de Baermann, que también permite observar otros parásitos además de Strongyloides. $38(84 \%)$ muestras fueron positivas al menos por un parásito. La prevalencia de nematodos fue Ascaris (36\%), uncinarias (22\%) Enterobius (4\%) y Trichuris (2\%). Para protozoos fue Endolimax nana (33\%), Entamoeba coli (27\%) y Entamoeba histolytica/dispar (11\%). Estos datos son un reflejo de las condiciones sanitarias inadecuadas de esta comunidad.

\section{REFERENCIAS}

1.- INCAP/OIR/MS. Evaluación nutricional de la población de Centro América y Panamá, Costa Rica. Instituto de Nutrición de Centro América y Panamá (INCAP), Oficina de Investigación Internacional (OIR), Ministerio de Salud de Costa Rica (MS), Publicación del INCAP, Guatemala, Guatemala. 1969.

2.- MATA L, PARDO V, FERNÁNDEZ F C. et al. Control and Erradication of Infectious Diseases. An International Symposium- PAHO copubl. Ser. No. 1, Pan American Health Organization, Washington, D.C., 1985.

3.- MATA L, HERNÁNDEZ F, PARDO V. Encuesta Nacional de Nutrición. Fascículo 5: Helmintos intestinales. Ministerio de Salud, Costa Rica. 1998.

4.- REYES L, MARÍN R, CATARINELLA G, et al. Parasitosis intestinal en niños de guarderías de San José, 
Costa Rica. Rev Cost Cienc Méd 1987; 8: 123-8.

5.- KOSOFF P, HERNÁNDEZ F, PARDO V, et al, Urban helminthiasis in two socioeconomically distinct Costa Rican communities. Rev Biol Trop 1989; 37: 181-6.

6.- HERNÁNDEZ F, LEÓN D, BRENES A, ROBINSON C. Parasitismo intestinal en un precario de San José, Costa Rica. Rev Cost Cienc Med 1998; 19: 245-7.

7.- CERDAS L. Parasitismo intestinal en Costa Rica. Tesis Licenciatura en Microbiología, Universidad de Costa Rica, Facultad de Microbiología 1999.

8.- HERNÁNDEZ-CHAVARRÍA F, AVENDAÑO L. A Simple Modification of the Baermann Method for
Diagnosis of Strongyloidiasis. Men Inst Oswaldo Cruz 2001; 96: 805-7.

9.- BOUZA-MORA L, RODRÍGUEZ-MASÍS D, HERNÁNDEZ-CHAVARRÍA F, MACHADO L. Strongyloides stercoralis en pacientes psiquiátricos. Rev Cost Cienc Méd. 2005 En prensa.

10.- AVENDAÑO L, HERNÁNDEZ F, JIMÉNEZ F, et al. Strongyloides stercoralis en pacientes alcohólicos. Parasitol al Día 1999; 23: 91-4.

11.- SÁNCHEZ A, MORA J R., HERNÁNDEZ F. Parásitos intestinales en pacientes de la tercera edad, Hospital Raúl Blanco Cervantes. Rev Cost Cienc Méd 1999; 20 : 167-73. 